\title{
FIGO Stage IB1
}

National Cancer Institute

\section{Source}

National Cancer Institute. FlGO Stage IB1. NCI Thesaurus. Code C96249.

A FIGO stage term that applies to gynecologic cancers. For cervical cancer, it refers to clinically visible lesions that measure $4.0 \mathrm{~cm}$ or less in diameter; there is no FIGO stage IB1 for endometrial cancer. 